\title{
Disrupting rock engineering concepts: is there such a thing as a rock mass digital twin and are machines capable of learning rock mechanics?
}

\author{
D Elmo University of British Columbia, Canada \\ D Stead Simon Fraser University, Canada
}

\begin{abstract}
Introduced by NASA engineers in the early 1960s during the Apollo space program, the term 'digital twin' has recently gained more visibility and exposure thanks to the development of the related new concept of the Internet of Things, IOT. A digital twin is not merely a model of a real physical asset but represents the actual connection between the physical world and the virtual (digital) reality. In this context, numerical models of a rock mass are virtual prototypes of that rock mass, which can be used to guide the design process and to estimate possible ground behaviour; they cannot however be considered true rock mass digital twins.
\end{abstract}

Rock engineering differs from other engineering disciplines since often design must be completed prior to developing access to rock exposures, forcing engineers and practitioners to rely on information obtained from severely limited 1D sampling methods. Even if engineers were to have unlimited resources, the natural variability of the rock mass, combined with the limited knowledge of the rock mass in the early phases of a project, would be such that the design outcome would still be influenced by what we do not know rather than by what we effectively know. The authors strongly believe that if initial 1D information to be complemented by $2 D$ sampling during slope excavation, and smart sensors embedded within the rock mass, the opportunity exists to update virtual models and to compare models to the data from the smart sensors on a periodic basis. Note that the smart sensors would have to provide more information than deformation alone, since failure of intact rock bridges in engineered slopes is a progressive damage process and as such, requires location of the source where damage is accumulating.

This paper outlines the challenges facing the rock engineering community if we really want to truly transform and improve virtual (digital) geological and geomechanically rock mass models. The authors also provide a critical discussion on the potential use of machine learning algorithms based on empirical methods and the use of qualitative to semi-quantitative scales of measurements that are inappropriate for a full statistical analysis.

Keywords: machine learning, digital twin, rock engineering

\section{Introduction}

When considering the development of rock engineering, Hoek (2007) observed that prior to the 1960s, several authors had worked on engineering projects that dealt with issues related to the stability of rock masses. This represents the first real phase in the application of rock engineering in practice. The evolution of the subject of rock mechanics saw a second phase in the application of rock engineering coinciding with the well-known and extensively studied Malpasset, France and Vajont, Italy dam disaster in 1959 and 1963 respectively. These catastrophic failures forced rock engineers to change the way we investigate rock masses to recognise the importance of the interaction of stresses, rock strength and geological structures. Rock mass classification systems introduced in the late 1960s and 1970s with the purpose of assigning numbers to geological information and developing an understanding of possible ground behaviour are a good example of the second phase in rock engineering applications with still these tools still widely used today. The use of numerical models has significantly increased in the past 20 years thanks to improved, more user-friendly and commercially available software along with more powerful computers. This so-called computer revolution 
led to a third phase on the evolution of the application rock engineering. We suggest that the more recent introduction of state-of-the-art ground monitoring instrumentation such as 'smart sensors' (e.g. smart markers planned for future block cave operations) with real time monitoring and better integration with other disciplines (e.g. CT scans used to study brittle failure processes) combined with a new generation of rock mechanics engineers who are highly knowledgeable about coding information into scripts supporting machine learning capabilities, represents a transition to a new or fourth phase in rock engineering applications in the mining industry.

The objective of this paper is to discuss several important issues that in the authors' opinion will impact and potentially constrain the introduction, success and acceptance of machine learning concepts in rock engineering. These issues include:

- The definition of a digital twin concept for rock engineering applications.

- The important role of uncertainty and variability along with the introduction of the concepts of close and open set uncertainties as applied to rock engineering data.

- The definition, applications, and limitations of machine learning methods, in the context of the empirical knowledge that is so important in conventional rock engineering design.

\section{The digital twin concept in rock engineering}

The original concept of the digital twin was introduced by NASA engineers in the early 1960s in the Apollo space program with NASA using a vehicle on Earth-identical to the one involved in the lunar mission-to reproduce the conditions that would be encountered in space by its digital space twin. With the development of the related new concepts of the Internet of Things (IOT), artificial intelligence (AI), and machine learning (ML), the digital twin concept has gained increased visibility and is now being recognised as important over a wide range of society including economists, entrepreneurs and engineers. The definition of a digital twin (Grieves 2003) includes not only the concept of replicating a physical object or a physical process, but more importantly, centres around the notion that a connection exists between the physical and digital objects such that the digital twin is continuously provided with data originating from the physical object. Thanks to the integration of ML algorithms, the digital twin can now learn from those data and generate simulations of the possible instances that the physical object may experience. In this context, numerical models of the rock mass used by engineers to guide the design process and to estimate possible ground behaviour although representing virtual prototypes of the rock mass do not however meet the definition of rock mass digital twin.

Figure 1 attempts to graphically define the concept of a digital twin and its components as applied to a rock engineering process. If the input data, including material properties, loading conditions and geometries, are either not expected to change, or their coefficients of variation do not result in a significant variability of the overall system, then the physical-to-digital link is circular, and the output data generated by monitoring the physical process can be used to forecast its behaviour, assess consequences of that behaviour and implement essential changes, which in turn will be monitored and analysed as part of the continuous cycle.

A discrete fracture network (DFN) model can be regarded as a virtual rock mass, and the connection between the physical and the virtual model improves as new data become available (Figure 2). Combined with a geocellular approach, the virtual rock mass represents the open discontinuities network and could be integrated with properties concerning intact rock strength, veinlets and incipient (cemented) joints to produce a rock mass grid model, with the grid resolution controlled by the representative elementary volume of the rock mass. Note that the DFN model would not meet the full definition of a rock mass digital twin unless a connection can be established by generating data in real time.

However, rock engineering differs from other engineering disciplines since design must often be completed prior to developing access to rock exposures, forcing engineers and practitioners to initially rely on information obtained from severely limited 1D sampling methods (Figure 3). Even if engineers had unlimited resources, the natural variability of the rock mass, combined with the limited knowledge of the rock mass in the early stages of a project, would be such that the design outcome would still be influenced by what we do 
not know rather than by what we know. The uncertainty and variability associated with natural rock materials is such that the assessment component in Figure 1 would require a second link, whereby either new input data are fed into the system as they become available, or the results of the analysis are used to define the amount and spatial location of new data being required to update the design. The gap between input data, design and decisions represents the knowledge gap. The larger this gap, the more data are required to bridge input data and design. The objective of the data collection process is to close that gap, or at least to reduce the reliance of the decision process on (subjective) engineering judgment and assumptions (see also Figure 3). These aspects will be further discussed in Section 3 below.

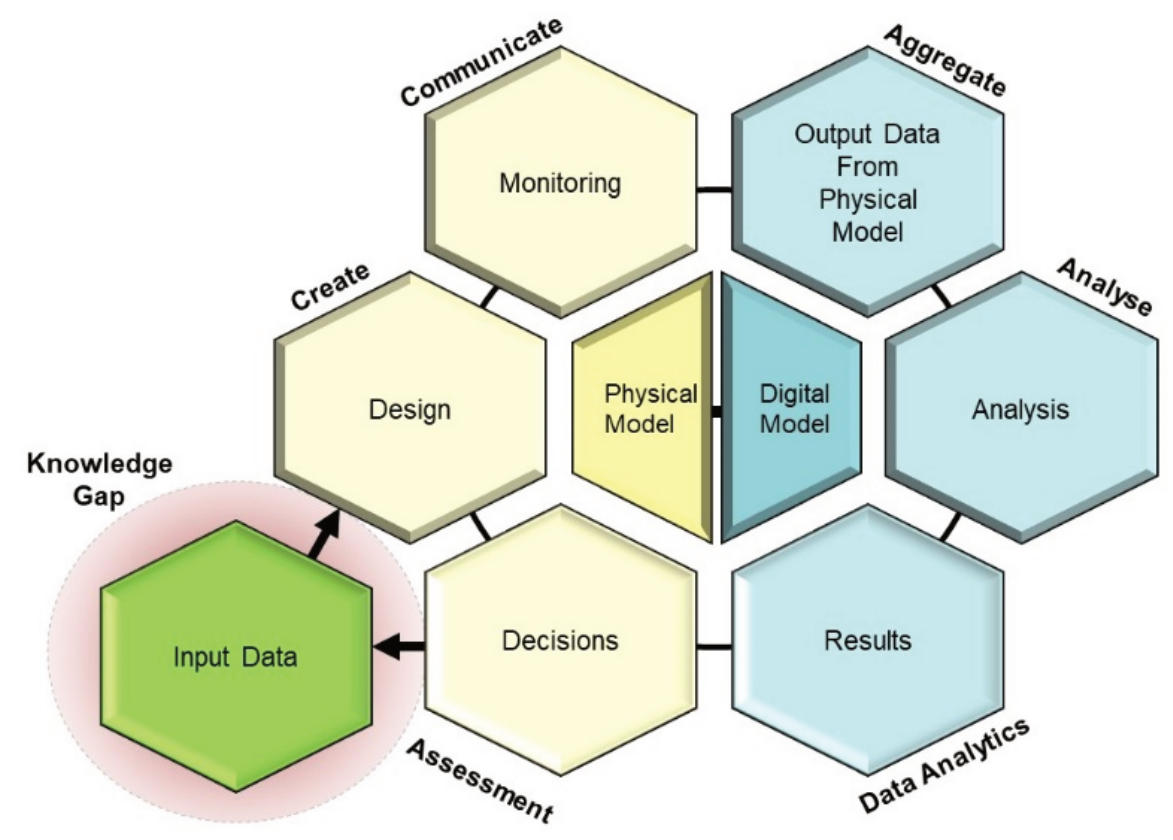

Figure 1 Concept of rock engineering digital twin

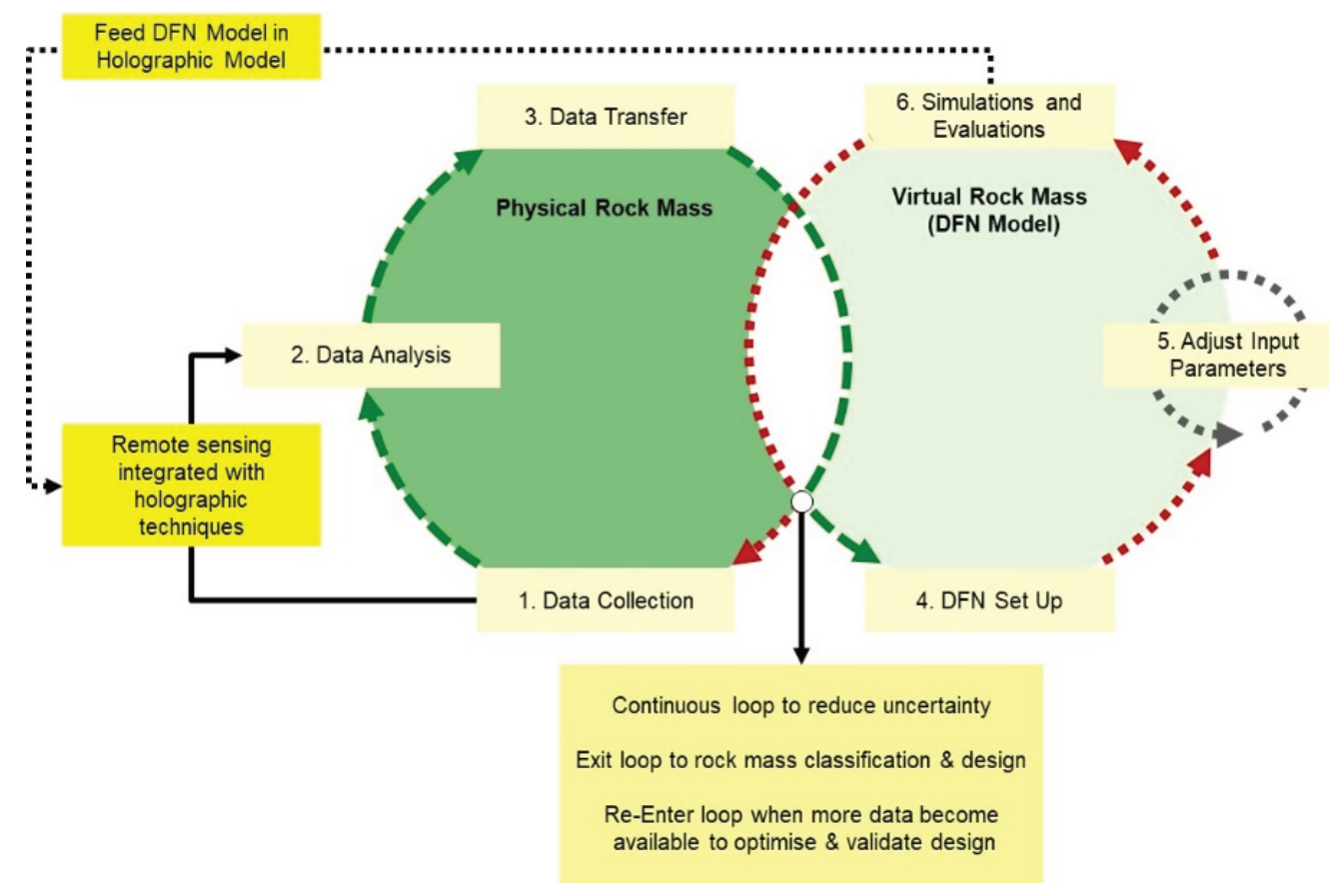

Figure 2 Virtual rock mass concept 
New methods of data acquisition offer the opportunity

to reduce uncertainty

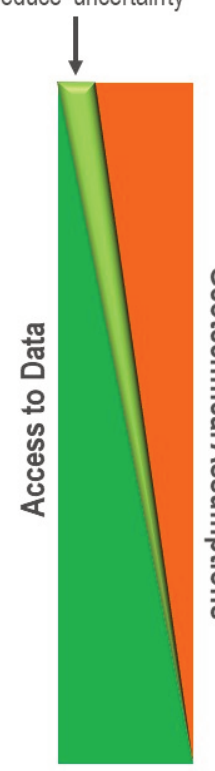

In rock engineering design often has to be completed prior to developing access to rock exposures

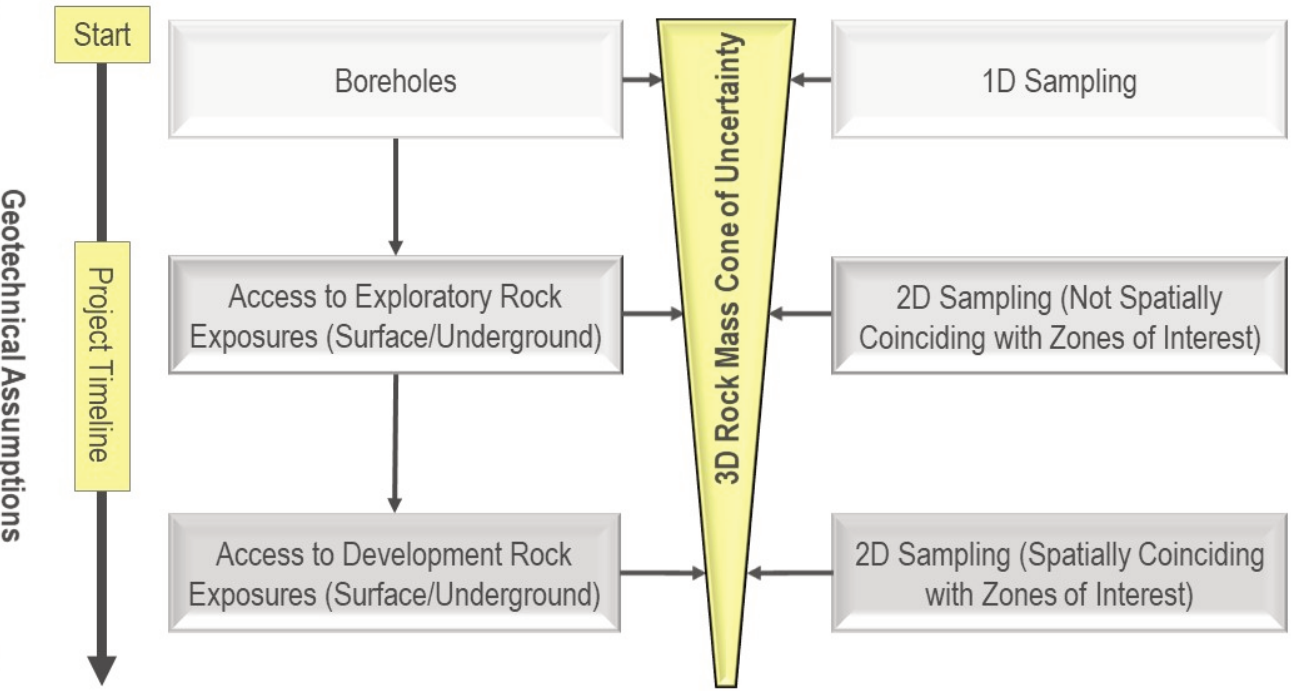

Figure 3 What makes rock engineering different from other engineering disciplines

\section{The role of uncertainty and variability in rock engineering design}

Rock engineering design, including slope stability analysis, is strongly linked to the concepts of uncertainty and variability (Mayer 2015). The randomness of natural geological processes is responsible for the manifested variability of rock material parameters. It is therefore important that we do not confuse variability (defined as the observable manifestation of heterogeneity of physical parameters and/or processes) with uncertainty (defined as relating to the engineer's state-of-knowledge, reflecting the decision to recognise and describe the observed variability in a qualitative or quantitative manner) (Uzielli 2008).

Uncertainty in geotechnical engineering is a multi-faceted problem which includes the geological, model, parameter and human uncertainties which can be defined as:

- Geological uncertainty: Randomness of geological processes dictates that the identification, characterisation and interpretation of the site geology and hydrogeology is not a linear problem.

- Parameter uncertainty: Due to the lack of, and/or insufficient knowledge of, key parameters, spatial variability in rock properties, and scale effects (e.g. intact rock versus rock mass properties).

- Model uncertainty: Inductive design approach and gaps in the scientific theory force engineers to make predictions on the basis of causal inference.

- Human uncertainty: Subjectivity and measurement error, differing professional opinions.

It would be safe to conclude that our knowledge of the rock mass would progressively increase by drilling more and more boreholes, excavating exploratory drifts and/or exposing surface outcrops. However, such a conclusion does not necessarily imply that the added knowledge would contribute to reducing uncertainty. Building on the discussion by Taleb (2010), a new geometric terminology can be introduced to define two groups of uncertainty (Figure 4):

1. Closed set uncertainty: This occurs when no single instance will significantly change the aggregate of a large enough sample. That is, if the sample is large enough, the aggregate knowledge will not change by adding more data points. In terms of rock engineering, this definition implies that we should not expect a random event to dominate a phenomenon (e.g. the stability of a slope) as long as we have reached a critical state-of-knowledge of the rock mass. Any additional knowledge would 
not change the outcome of the engineering design; our assessment of the probability of failure would not change, and the risk associated with a potential slope failure would only depend on varying the consequences of the slope failing.

2. Open set uncertainty: This occurs when one simple observation can disproportionally impact the aggregate knowledge. In this case, it is difficult to measure the average of a sample since it could be affected by one single data point. This condition may occur in slope stability analysis, if, for example, the data collection process did not reveal the presence of major structures or somehow overestimated the role of intact rock bridges. The toe-break out problem analysed by Stead et al. (2007) and Elmo et al. (2009) is a good example of an open set uncertainty, whereby the stability of a slope is determined by the combined knowledge of: i) existence of a major structure behind the slope face; and ii) the distance of the major structure from the slope face, all of which may be unknown.

\section{Complete} Ignorance

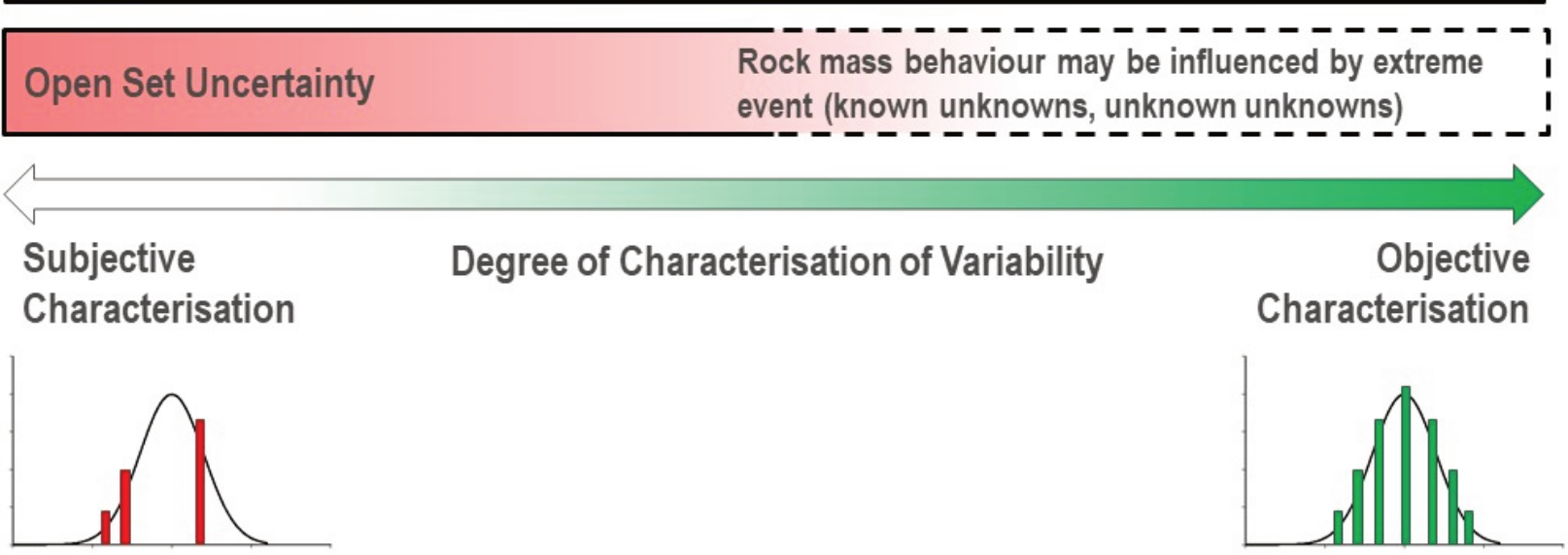

Figure 4 Relationship between knowledge, uncertainty (closed and open set) and variability

In the context of the above definitions and considering the components of geotechnical slope design (bench, inter-ramp and overall slope stability, respectively), uncertainty can also be considered as a scale dependent phenomenon (Figure 5). For a given problem, total uncertainty would be the summation of closed and open set forms of uncertainties. The larger the problem scale, the greater the knowledge required to reduce open set uncertainty, and thus reduce the risk of an extreme event occurring. Bench design is typically carried out by assuming continuous and ubiquitous joints, thus intact rock bridges can only be accounted for in an implicit manner. Therefore, joint orientation and shear strength of the joint surfaces are the only uncertainties present. Bench face angle is not considered an uncertainty since it represents a known engineered controlled variable. Joint orientation bias could be corrected using Terzaghi or Mauldon procedures. Note that the Guidelines for Open Pit Slope Design (Read \& Stacey 2009) define 25\% to 50\% probability of failure as an acceptable limit for bench design. In this context, there may not be any advantage in adding more data and the design may, to some extent, already account for extreme events by using a relatively high probability of failure for design acceptability. Under those conditions, both joint orientation and joint shear strength represent a form of closed set uncertainty, since adding more knowledge to the aggregate sample would not necessarily alter the outcome of the analysis.

The procedure of adopting conservative assumptions can be considered as a method to interpret rock mass behaviour by bridging the knowledge gap with arbitrary knowledge. In the case of continuous and ubiquitous 
joints for example, we create an artificial extreme event and design for it. However, when it comes to overall slope stability, the assumption of fully continuous and ubiquitous joints is geologically not acceptable. Therefore, overall slope design is controlled by open set uncertainty since the design may not have necessarily accounted for all possible extreme events. In other words, overall slope design is more likely to be impacted by unforeseen conditions, whether in the form of 'known unknowns', or in the form of 'unknown unknowns'.

Correct consideration of uncertainty types, limited knowledge and poor characterisation of geological variability would all inevitably impact the accuracy and utility of ML predictions.

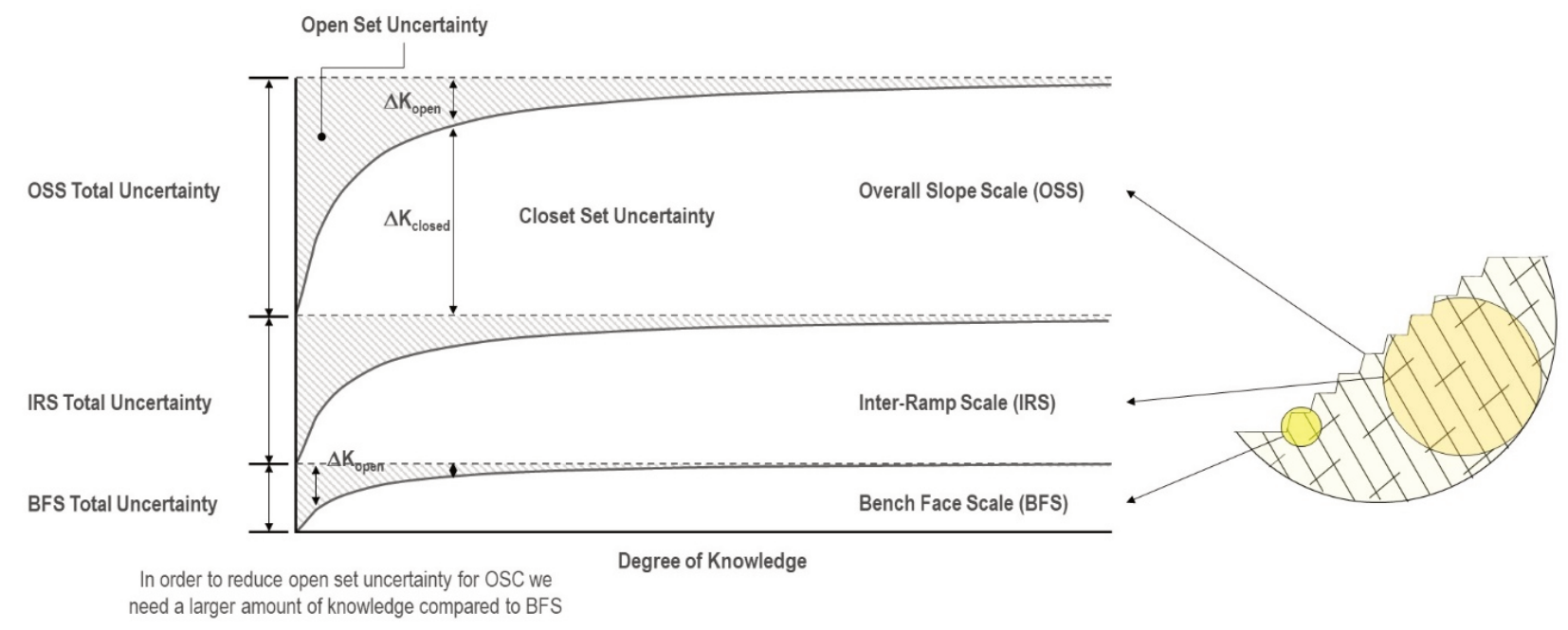

Figure 5 Uncertainty and scale effects (BFS = bench face scale, IRS = inter-ramp scale, and OCS = overall slope scale)

\subsection{Rock bridges and other important known unknowns in open pit slope stability}

Characterisation and analysis of intact rock bridges has been the focus of considerable research, dating back to Terzaghi (1962) and Jennings (1970), who first discussed the role of intact rock bridge failure in the context of rock slope stability. Other researchers have investigated intact rock bridges and the associated problem of step-path failure including Call \& Nicholas (1978), Einstein et al. (1983), Read \& Lye (1984), Baczynski (2000, 2008), and Dershowitz et al. (2017). Elmo et al. (2018) showed that there are still many unanswered questions, beginning with the definition of an intact rock bridge itself (Figure 6). Field experiments by Hencher et al. (2012) and Shang et al. (2017) have clearly demonstrated that rigorous in-plane rock bridges measurements are possible only after failure has occurred. Clearly, this represents a major obstacle for design scenarios, since it implies that it is not possible to validate assumptions made with respect to the extent of in-plane rock bridges without performing field testing or back-analysis. Direct measurements of down-slope or lateral rock bridges would be even more complicated, since their definition is linked to more than just geometrical arrangements between joints. According to Elmo et al. (2018), it is not possible with current technology to provide exact locations and measurements of rock bridge percentages (and failure paths) pre-failure. The same authors recognised that probabilistic methods such as DFN modelling could help in providing a mode of rock bridge measurement; however, DFN modelling alone would be insufficient since rock bridge failure is a rock mass damage process and therefore rock bridge strength analysis is more complicated than then simple formulation as proposed by Jennings (1970). In this context, rock bridges represent a clear example of 'known unknowns', and their impact on slope stability analysis would be defined by the rules of open set uncertainty.

The definition of a rock bridge is directly related to the definition of joint length. Joint length may initially be considered as a 'known unknown' (before access to surface outcrops is possible), becoming a 'known known' once, for example, a slope is being excavated. The definition and measurement of joint length requires geotechnical engineers be familiar with truncation and censoring biases (i.e. values below a certain joint 
length are omitted (truncation) or relatively larger values cannot be measured because of the limited extent of the rock exposure (censoring)).

Joints termination is another parameter that is important in rock mass behaviour, since it provides a hierarchal structure to relate joint sets, it defines rock mass blockiness and indirectly rock bridges. However, information on termination types are seldom collected in the field, and they cannot be quantified from 1D sampling; therefore, joint terminations can be considered as 'known unknowns' and an open form of uncertainty.

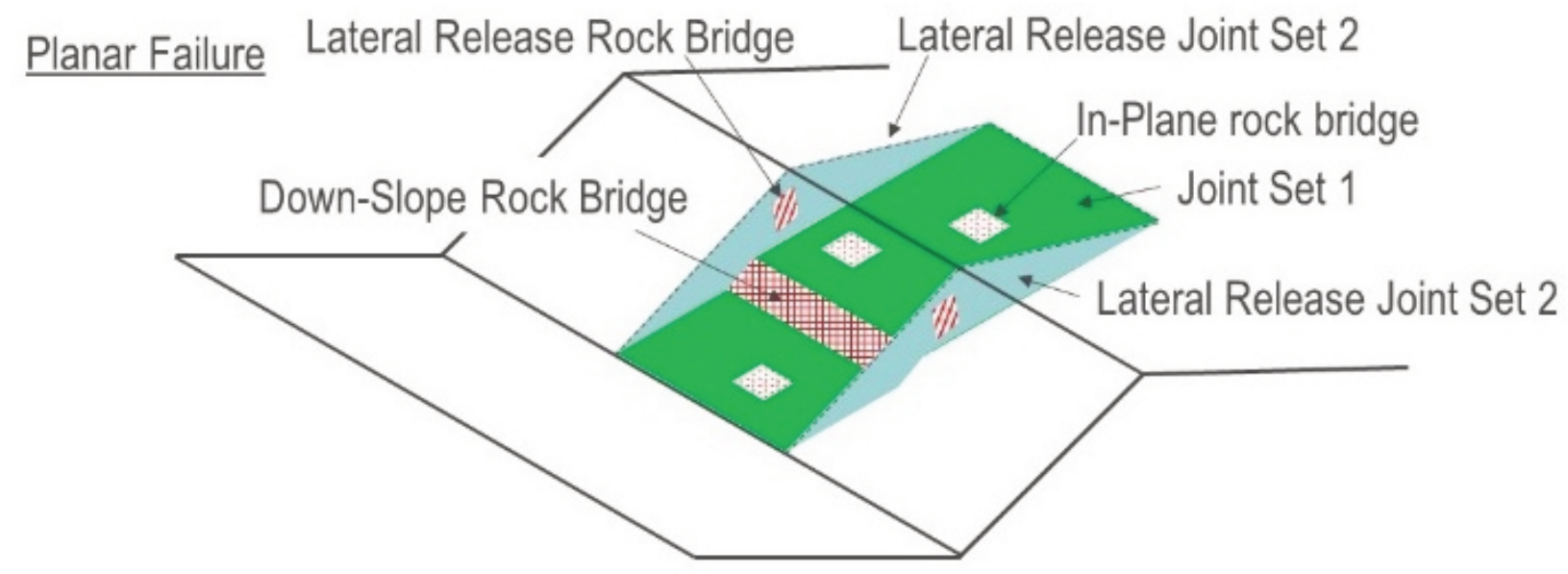

(a)

\section{Wedge Failure In-Plane Rock Bridge}

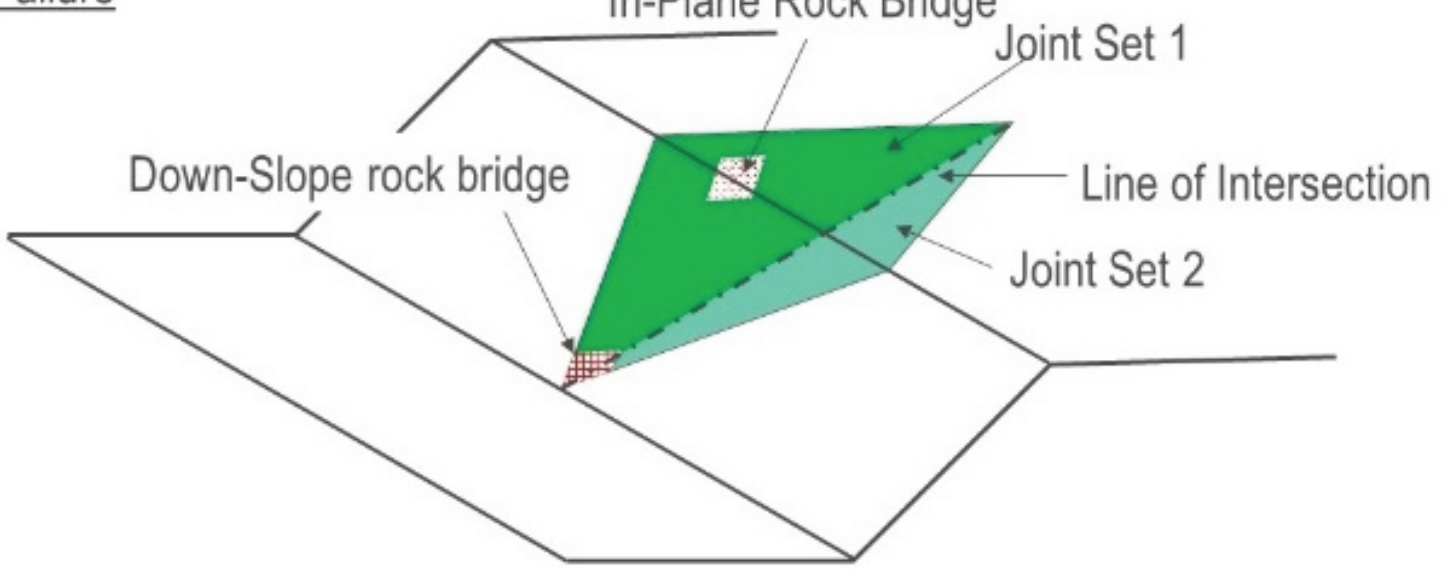

(b)

Figure 6 Definition of intact rock bridges for (a) planar and (b) wedge failure (modified from Elmo et al. 2018)

\subsection{Engineering judgment and human uncertainty}

Based on the previous discussion, geotechnical engineers typically work under the conditions of limited information. If we now paraphrase the discussion provided in Taleb (2010) and compare the two statements below:

1. There is no evidence of slope failure when considering current monitoring data.

2. There is evidence of no slope failure when considering current monitoring data.

At first, the two sentences may appear to support the same conclusion, however, when looking more closely, it is clear the two statements refer to very different conclusions. According to Statement 1, data are used to conclude that the slope is stable, however, the occurrence of an instance that could lead to failure cannot be 
excluded a priori and may be confirmed by collecting more monitoring data. Statement 2 , however, excludes slope failure in absolute terms, since it claims that there is evidence supporting the conclusion of no failure. Engineers should be very careful when invoking data as evidence to eliminate outcomes, since data represents a degree of knowledge, but it is not necessarily the case that data uncertainty is of the closed form, in which case extreme events cannot be ruled out.

The same author (Taleb 2010), claimed that "Knowledge, even when exact, may not lead to appropriate actions". This comment is clearly appropriate with respect to applications of $\mathrm{ML}$, since the human mind works based on data interpretation and reduction. Therefore, engineering judgment may be biased, as our mind subconsciously interprets data by confirming instances that agree with our assumed knowledge of a phenomenon and excludes data that either do not agree with our assumptions or may add uncertainty. The term 'industry standard' is often used in rock engineering when referring to empirical methods (e.g. rock quality designation (RQD), Deere et al. 1967) generally accepted by industry. However, the definition of industry standard does not imply that those methods are the best technical solutions, nor does it imply the methods are correct. For instance, it is true that empirical methods are not always wrong, but it is not true that empirical methods are always correct. Under these circumstances, what would be the implication of expressing the accuracy of a ML algorithm attempting to recognise rock mass classification patterns? This discussion may sound academic and philosophical; however, being able to understand the nature of human uncertainty is fundamental when integrating $\mathrm{ML}$ algorithms to help with rock stability design.

\subsection{Levels of measurement: qualitative versus quantitative measurements}

The levels of data measurements and the methods adopted for data collection and data characterisation, govern the appropriate type of statistical analysis geotechnical engineers should apply to assess slope stability analysis. According to Stevens (1946), there four different level of measurements can be recognised in descending order of precision:

- Nominal: words, letters and alphanumeric symbols can be used to classify data.

- Ordinal: the data measurement represents an ordered relationship among the variables' observations.

- Interval: equal distances along a measurement scale (intervals) can be used not only to classify and order measurements, but also to compare them.

- Ratio: an interval measurement for which the variables' observation could coincide with an absence of the quantity being measured (i.e. an interval measurement for which zero is permitted).

Each scale is strictly limited to specific statistical operations; interval and ratio scales allowing for the calculation of means and standard deviations, whereas nominal and ordinal measurements are limited to medians and percentiles (Harrison 2017). Rock mass classification systems make use of either nominal or ordinal measurements, whereby properties such as fracture spacing, or number of fracture sets are interpreted based on empirically defined ranges in order to provide a rating number. For example, in the rock mass rating system (Bieniawski 1989) a fracture spacing of $0.6 \mathrm{~m}$ would be assigned a rating of 15; but the same rating could also be assigned to any fracture set with a spacing between 0.6 and $2 \mathrm{~m}$. Some engineers may contend that there some degree of flexibility is needed, and one should adopt some form of engineering judgment when selecting the ratings. However, the use of engineering judgment cannot solve the issue with the ordinal nature of the measurement that is being made. The method commonly used for field classification of intact rock strength is another example of a nominal scale measurement, whereby rock strength is estimated based on the number of blows required to break or indent a piece of core.

The limitation of using either a nominal or ordinal scale in rock engineering is a combined form of parameter and model uncertainties. These are generally ignored by geotechnical engineers, since the process of assigning numbers to geology produces the misconception that, for example, classification ratings are a direct measurement of rock mass physical properties, which can in turn be analysed statistically. The use of parameters based on interval and ratio scales (e.g. fracture frequency) is therefore required if we really want 
to implement a more objective approach to address complex rock engineering problems and be able to successfully and meaningfully train ML algorithms. This will be further discussed in Section 4.

\section{$4 \quad$ Machine learning principles and rock engineering applications}

Applications of $\mathrm{ML}$ in a wide range of industries is ubiquitous in current practice and the mining industry is surely not immune to the pitfalls of using Al inappropriately. One may argue that $\mathrm{ML}$ is an attractive and appealing umbrella definition, under which it is possible to locate several algorithms with complex and media unfriendly terminology, including but not limited to, naive Bayes, random forest, artificial neural networks and support vector machines. A simple literature search quickly confirms that $\mathrm{ML}$ is not a new approach. Zhang \& Song (1991) discussed how neural networks could be applied to rock mechanics using either quantitative or qualitative data. The same authors pointed to the importance of training the neural network used to predict the behaviour of the rock mass. What has made ML more appealing in recent years is the wide availability of faster computers, high performing graphical processing units and open source deep learning libraries like TensorFlow.

In simple terms $\mathrm{ML}$ uses a trained dataset to recognise patterns, which are in turn used to make predictions or infer behaviour without the need for human intervention. This is the principle behind for example the use of $\mathrm{ML}$ for autonomous trucks in mining operations, ore sorting and mineral exploration. In this context, ML could be described as substitute for the human mind when completing specific tasks. However, things are not necessary as straightforward when it comes to using ML for engineering design.

Recent applications of ML in rock engineering include Bilal et al. (2018), who used ML to predict physical rock mechanical properties (e.g. Young's modulus) from well log data, rock mineralogy and texture. The authors' objective was to overcome the limitations of empirical relationships between dynamic and static mechanical properties of rock material. Similarly, Xu et al. (2019) have applied supervised ML to predict the penetration rate of tunnel boring machines, as an alternative to using empirical and theoretical techniques. The same authors provide a review of different ML techniques applied to study tunnel boring machine (TBM) performance since 2000. Ren et al. (2018) used a variety of ML algorithms to estimate intact rock compressive strength in the field using the acoustic characteristics of hammering sound samples. The predictions were then used as input in rock mass classification systems. Rockbursts is another subject which has be a subject of significant ML research. For example, Li et al. (2017) have studied rockburst prediction based on generic algorithms and extreme event ML. Zhou et al. (2016) have used a gradient boosting machine approach to develop a method to predict failure in slopes using of a relatively large database (221 cases). However, the method is limited to circular failure and the database is composed mostly of landslides. Maximum slope height in the database is $480 \mathrm{~m}$ for an unknown location.

ML algorithms require experimental databases, based on field observations and/or laboratory tests. Data are required to train the algorithm and to validate its predictions. However, one aspect that may not be highlighted in the literature, is the need to update the training of the algorithm using new data as they become available. It is reasonable to expect that the accuracy of the prediction would increase with increasing the amount of data. However, the validity of the above statement is a function of the specific ML algorithms being used; for instance, Pu et al. (2019) have shown that the accuracy of rockburst predictions may decrease when considering a larger database in combination with specific $M L$ methods such as adaptive network-based fuzzy inference system and Huberized support vector machine.

In Xu et al. (2019) the database used to train the ML algorithm included uniaxial compressive strength, Brazilian indirect tensile strength, RQD, weathering zone, thrust force, and revolution per minute of a TBM to predict penetration rate. RQD is a quantitative rock mass index, with known orientation bias and it depends on user-defined (subjective) threshold values. Weathering is also mostly characterised based using qualitative scales of measurement.

The lack of quantifiable variables, data uncertainty, and spatial variability of geological parameters create a condition under which is difficult to forecast time and location of a phenomenon. For example, when applied to rock bridge analysis, ML would have to solve the problem of the 'observer effect', that is the observation 
and measurement of a phenomenon (e.g. rock bridges) are associated with a change in the phenomenon being measured, since the physical evidence of rock bridges is only visible post-failure (Elmo et al. 2018). In the context of rockburst and rockfalls, McGaughey (2019) noted another major limitation of ML algorithm that is the condition to be forecasted often depends on factors inferred from models that are themselves created from sparse measurement or drillhole data. The same author argued that ML is a method that offers the ability to look for patterns and correlations, and not a method that can on its own describe a new physical model of rock mass behaviour. The concept echoes Marcus (2017), who called ML a "passive dredging system" to help finding new correlations between datasets. When considering different phases in a rock engineering design project (Figure 7), ML therefore becomes a method that concentrates data by filtering those through new correlations and patterns. This filtering process should hopefully reduce the transfer of uncertainty between the different rock engineering phases.

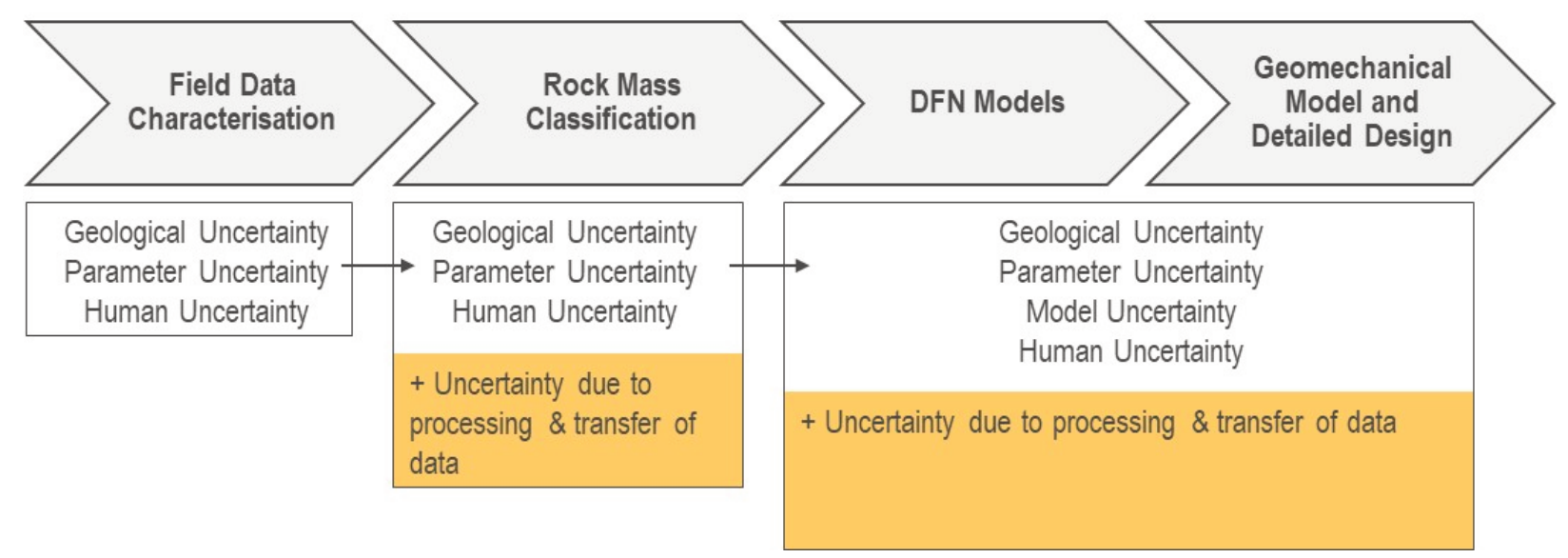

Figure 7 Typical phases in a rock engineering design project

\section{Conclusion}

This paper has presented a discussion on the introduction of important concepts such as the digital twin and $M L$ in rock engineering. $M L$ is no longer confined to the world of academia and machine learning models can now be easily built within easy to use platforms (e.g. Python and MATLAB coding). The terminology 'extreme learning' and 'deep learning' have begun to evoke a sense of accuracy and infallibility that, however, is in direct contrast with our current state-of-knowledge of rock mechanics phenomena. This paper deliberately focuses on academic but fundamental arguments that are at the core of ML. It would be all too tempting and unwise to mistake $M L$ as a solution to all rock engineering design problems rather than another (but very effective) instrument in the geotechnical engineers' toolbox. Like any geotechnical tool, ML has advantages and limitations; the former can only be fully exploited by learning the latter.

\section{Acknowledgement}

The author would like to acknowledge the financial support through the Natural Sciences and Engineering Research Council of Canada grants hold by Dr Davide Elmo.

\section{References}

Baczynski, NRP 2000, 'Stepsim4 - Step-path method for slope risks', Proceedings of the International Conference on Geotechnical and Geological Engineering, Melbourne.

Baczynski, NRP 2008, 'STEPSIM4 Revised: network analysis methodology for critical paths in rock mass slopes', in Y Potvin, J Carter, A Dyskin \& R Jeffrey (eds), Proceedings of the First Southern Hemisphere International Rock Mechanics Symposium, Australian Centre for Geomechanics, Perth, pp. 405-418.

Bieniawski, ZT 1989, Engineering rock mass classification, Wiley, New York. 
Bilal, S, Negara, A \& Shujath, S 2018, 'Digital rock physics combined with machine learning for rock mechanical properties characterization', Proceedings of the Abu Dhabi International Petroleum Exhibition \& Conference, Society of Petroleum Engineers, Abu Dhabi.

Call, RD \& Nicholas, DE 1978, 'Prediction of step path failure geometry for slope stability analysis', Proceedings of the 19th US Symposium on Rock Mechanics, Mackay School of Mines, Reno.

Deere, DU, Hendron, AJ, Patton, FD \& Cording, EJ 1967, 'Design of surface and near surface construction in rock', in C Fairhurst (ed.), Proceedings of the 8th U.S. Symposium on Rock Mechanics-Failure and Breakage of Rock, American Institute of Mining, Metallurgical and Petroleum Engineers, Inc., New York, pp. 237-302.

Dershowitz, WS, Finnila, A, Rogers, S, Hamdi, P \& Moffitt, KM 2017, 'Step path rock bridge percentage for analysis of slope stability', Proceedings of the 51st U.S. Rock Mechanics/Geomechanics Symposium, American Rock Mechanics Association, Alexandria.

Einstein, HH, Veneziano, D, Baecher, GB \& O'Reilly, KJ 1983, 'The effect of discontinuity persistence on rock slope stability', International Journal of Rock Mechanics and Mining Sciences \& Geomechanics Abstracts, vol. 20, pp. 227-236.

Elmo, D, Donati, D \& Stead, D 2018, 'Challenges in the characterization of rock bridges', Engineering Geology, vol. 245, pp. 81-96.

Elmo, D, Moffitt, K, D'Ambra, S \& Stead, D 2009, 'A quantitative characterisation of brittle rock fracture mechanisms in rock slope failures', in JR Read (ed.), Proceedings of the 2009 International Symposium on Rock Slope Stability in Open Pit Mining and Civil Engineering, University de los Andes, Santiago.

Grieves, J 2003, Strategic Human Resource Development, SAGE Publications Ltd, London, DOI: http://dx.doi.org/10.4135/ 9781446216859

Harrison, J 2017, 'Rock engineering design and the evolution of Eurocode 7', Proceedings of the EG50 Engineering Geology and Geotechnics Conference, International Society of Rock Mechanics, Portsmouth.

Hencher, SR, Lee, SG, Carter, TG \& Richards, LR 2012, 'Sheet joints: Characterization, shear strength and engineering', Rock Mechanics and Rock Engineering, vol. 44, pp. 1-22.

Hoek, E 2007, Practical rock engineering, RocScience, Vancouver, https://www.rocscience.com/assets/resources/learning/ hoek/Practical-Rock-Engineering-Full-Text.pdf

Jennings, JE 1970, 'A mathematical theory for the calculation of the stability of slopes in open cast mines', in PWJ Van Rensburg (ed.), Proceedings of the Symposium on the Theoretical Background to the Planning of Open Pit Mines, South African Institute of Mining and Metallurgy, Cape Town, pp. 87-102.

$\mathrm{Li}, \mathrm{T}$, Lee, X \& Xiao-li, Y 2017, 'Rock burst prediction based on genetic algorithms and extreme learning machine', Journal of Central South University, vol. 24, issue 9, pp. 2105-2113.

Marcus, G 2017, Artificial Intelligence is Stuck. Here's How to Move it Forward, New York Times, New York.

Mayer, J 2015, Applications of uncertainty theory to rock mechanics and geotechnical mine design, MSc thesis, Simon Fraser University, Burnaby.

McGaughey, J 2019, 'Artificial intelligence and big data analytics in mining geomechanics', in W Joughin (ed.), Proceedings of the Ninth International Conference on Deep and High Stress Mining, The Southern African Institute of Mining and Metallurgy, Johannesburg, pp. 45-54.

$\mathrm{Pu}, \mathrm{Y}, \mathrm{Apel}, \mathrm{D}, \mathrm{Liu}, \mathrm{V}$ \& Mitri, H 2019, 'Machine learning methods for rockburst prediction-state-of-the-art review', International Journal of Mining Science and Technology, vol. 29, issue 4, pp. 565-570.

Read, J \& Stacey, P 2009, Guidelines for Open Pit Slope Design, Taylor \& Francis Ltd, London.

Read, JR \& Lye, GN 1984, 'Pit slope design methods: Bougainville copper open cut', Proceedings of the 5th International Congress on Rock Mechanics, Rotterdam: AA Balkema, Melbourne, pp. C93-C98.

Ren, Q, Han, S \& Lin, M 2018, Prediction of rock compressive strength using machine learning algorithms based on spectrum analysis of geological hammer', Geotechnical and Geological Engineering, vol. 37, issue 1, pp. 475-489, 'https://doi.org/ 10.1007/s10706-018-0624-6

Shang, J, Hencher, SR, West, LJ \& Handley, K 2017, 'Forensic Excavation of Rock Masses: A Technique to Investigate Discontinuity Persistence', Rock Mechanics and Rock Engineering, vol. 50, pp. 2911-2928.

Stead, D, Elmo, D, Yan, M \& Coggan, J 2007, 'Modelling brittle fracture in rock slopes: experience gained, and lessons learned', in Y Potvin (ed.), Proceedings of the 2007 International Symposium on Rock Slope Stability in Open Pit Mining and Civil Engineering, Australian Centre for Geomechanics, Perth, pp. 239-252.

Stevens, SS 1946, 'On the theory of scales of measurement', Science, vol. 103, issue 2684, pp. 677-680.

Taleb, N 2010, The Black Swan: The Impact of the Highly Improbable, Random House, 400 pp.

Terzaghi, K 1962, 'Stability of steep slopes on hard unweathered rock', Géotechnique, vol. 12, pp. 251-270.

Uzielli, M 2008, 'Statistical data of geotechnical data', in AB Huang \& PW Maybe (eds), Geotechnical and Geophysical Site Characterisation, CRC Press, London.

Xu, H, Zhou, J, Asteris, PG, Armaghani, DJ \& Tahir, MM 2019, 'Supervised Machine Learning Techniques to the prediction of tunnel boring machine penetration rate', Applied Science, vol. 9.

Zhang, Q \& Song, J 1991, 'The application of machine learning to rock mechanics', Proceedings of the 7th ISRM Congress, International Society for Rock Mechanics and Rock Engineering, Aachen.

Zhou, J, Li, X \& Mitri, H 2016, 'Classification of Rockburst in Underground Projects: Comparison of Ten Supervised Learning Methods', Journal of Computing in Civil Engineering, vol. 30, issue 5. 
Disrupting rock engineering concepts: is there such a thing as a rock mass digital twin and are machines capable of learning rock mechanics? 